\title{
Quantum Kinetic Theory for a Condensed Bosonic Gas
}

\author{
R. Walser, J. Williams, J. Cooper and M. Holland \\ JILA, National Institute for Standards and Technology and University of Colorado, Boulder, CO 80309-0440
}

(February 16., 1999)

\begin{abstract}
We present a kinetic theory for Bose-Einstein condensation of a weakly interacting atomic gas in a trap. Starting from first principles, we establish a Markovian kinetic description for the evolution towards equilibrium. In particular, we obtain a set of self-consistent master equations for mean fields, normal densities, and anomalous fluctuations. These kinetic equations generalize the GrossPitaevskii mean-field equations, and merge them consistently with a quantum-Boltzmann equation approach.
\end{abstract}

PACS Nos. 03.75.Fi, 05.30.Jp, 42.55.Ah, 05.70.Ln

\section{INTRODUCTION}

With the experimental realization of Bose-Einstein condensation with neutral atomic gases [1] 3], it has become possible to observe fundamental properties of quantum statistics directly. While there are numerous wellestablished fields of low-temperature quantum physics that deal with many-particle systems, most of the salient features related to the paradigm of indistinguishablility are masked by strong interactions, or contact with thermalizing reservoirs. The newly gained ability to isolate the condensate fraction from its environment to a high degree, as well as the tight spatial confinement of the macroscopic quantum field, has opened up ways to selectively manipulate the mean-field, and study its temporal evolution towards equilibrium almost "in vivo".

The motion of trapped atoms in a dilute gas consists of free oscillations within the external potential that are interrupted by short binary collision events. Conventionally, that interaction strength is measured by the range of the repulsive two-particle potential, i.e. the scattering length $a_{\mathrm{s}}$. The duration of a collision event $\tau_{0}$ is given by the time a particle of average velocity $v$ spends in the interaction region, i.e., $\tau_{0}=a_{\mathrm{s}} / v$. On the other hand, the inverse collision rate $\tau_{\mathrm{c}}=1 /\left(n a_{\mathrm{s}}^{2} v\right)$ is an estimate of the time between successive collisions where $n$ denotes a particle density. As we are interested in low kinetic energies and the weak interaction limit, one finds a characteristic separation of time scales, i.e.,

$$
\tau_{\mathrm{c}} \gg \tau_{0}, \quad \text { or } n a_{\mathrm{s}}^{3} \ll 1 .
$$

It is this separation of time-scales that gives raise to a kinetic stage of evolution, preceding any equilibrium situation. For example, such a kinetic stage is absent in strongly interacting systems where a local equilibrium is established immediately ( $\tau_{\mathrm{c}} \approx \tau_{0}$, hydro-dynamic stage). Any individual collision event creates a quantum mechanical entanglement between collision partners. However, due to the long separation between successive collisions and the presence of intermediate weak fluctuations these temporal correlations decay rapidly (Markov approximation).

Based on this microscopic picture of the weakly interacting bosonic gas, we derive a generalized kinetic theory for a coarse-grained Markovian many-particle density operator, as discussed by A. I. Akhiezer and S. V. Peletminskii, [4. The quint-essential assumption behind this approximation is a coarse-grained density operator that depends only on a few selected variables. These quantities will serve as master variables and determine the system's evolution on a coarse-grained time scale. From a perturbative expansion of this density operator, we obtain kinetic equations that describe the temporal evolution of the expectation value of any (single-time) observable. As a specific application of this formulation, we then assume that the condensed gas can be described essentially by the dynamic evolution of mean fields, normaland anomalous fluctuations. In particular, we obtain kinetic equations that comprise the Gross-Pitaevskii equation and the quantum-Boltzmann equation as special instances. These kinetic equations are formulated basisindependently and consider all second-order processes which give raise to collisional energy shifts and damping rates. It is also noteworthy that the present theory can be applied readily to multi-component bosonic gases, provided the two-particle scattering matrix is interpreted accordingly.

However, considering the rapid development of this field of physics, it is also of great importance to compare the predictions of this and other kinetic theories 15 (mean-field equations and kinetic theories) with the results obtained from finite-temperature calculations based on the Hartree-Fock-Bogoliubov formulation [16 25] (collective excitation frequencies and damping rates), direct approaches to solve the many-particle Schrödinger equation 26.27] (configuration interaction, hyper-spherical coordinates), renormalization group techniques [28], and, above all, to gauge them against physical reality [29 31 . Further references on the extensive literature can be found in recent review articles and literature compilations 3234 .

The present article is organized as follows: In Sec. IIA, we introduce a coarse-grained statistical density operator and derive an integral equation for it in Sec. IIB. Assuming a weak two-particle collision rate, permits the 
development of a perturbation theory, which is described in Sec. II . From an explicit series expansion of the coarse-grained density operator, we obtain in Sec. IID a set of kinetic equations characteristic of a condensed bosonic gas. In Sec. IIIA, we introduce the Hamilton operator that governs the kinetic evolution of a weakly interacting, repulsive gas. A set of relevant operators is introduced in Sec. III B, i.e., macroscopic mean-fields, normal fluctuations, as well as anomalous averages. By applying the general kinetic master equations to this specific set of relevant operators, we obtain self-consistent kinetic master-equations and give a detailed discussion in Sec. III G. Finally, in Sec. IV, we discuss the prospects and possible implications for a numerical simulation of a fully quantum mechanical self-consistent solution.

\section{A COARSE-GRAINED MARKOVIAN DENSITY OPERATOR}

The quantum mechanical state of a many-particle system contains an overwhelming amount of data and it is the objective of quantum statistical mechanics to extract the relevant information from a data subset as small as possible. Such a minimal description then leads to a coarse-grained picture of the behavior of the system. The effects of coarse-graining on the dynamical evolution are well known from the quantum mechanics of open systems 35,36. By discarding some observable information from the unitary evolution of the complete system, one breaks the time-reversal symmetry, thus introducing irreversibility. However, a judicious partitioning of the many, interacting degrees of freedom of a large system into a small relevant subsystem and a weakly coupled, complementary reservoir leads to a tractable description of the subsystem's evolution towards equilibrium. This equilibrium state is determined by the properties of the reservoir.

In this section, we will pursue these general ideas and define a coarse-grained statistical density operator that depends functionally on only a few fundamental variables, and thus gives a raw picture of the "true" state of the many-particle system. Based on the definition for the coarse-grained statistical density operator, we then derive an integral equation that determines the functional form of this operator. In the limit of weakly interacting, dilute quantum gases where strong collisional interaction events are well separated in time, it is possible to solve this equation perturbatively and establish a hierarchy in terms of an expansion parameter proportional to this interaction strength. Once a perturbative expression for the functional form of the coarse-grained statistical operator is determined, we then use it to study the motion of expectation values of general observables. These equations of motion establish a closed, self-consistent set of kinetic equations for the following restricted set of fundamental variables: the mean field, the normal singleparticle density, and the anomalous fluctuations. By this method, we generalize the Gross-Pitaevskii mean-field equation and merge it consistently with an approach that leads to the quantum-Boltzmann equations for both normal densities and anomalous fluctuations.

In many ways our approach is reminiscent of the classical Bogoliubov-Born-Green-Kirkwood-Yvon (BBYGK) method 37], for in a similar manner, higher order correlations can also be characterized by a restricted set of variables. Specifically, in classical Markovian systems higher order correlation functions can be expressed in terms of single-particle densities.

\section{A. Fundamental assumptions of statistical mechanics}

The derivation of a coarse-grained density operator relies on the basic assumption of statistical mechanics that any non-equilibrium statistical correlation eventually decays [4,37 40]. For example, in classical kinetics, this time is typically of the order of the duration of a collision.

In the following discussion, we introduce three distinct many particle density operators. The first density operator, $\rho(t)$, describes the complete state of the manyparticle system. Starting from an initial value $\rho(0)$, the system evolves unitarily in time according to a time independent Hamilton operator $\widehat{H}$. In analogy with the celebrated Chapman-Enskog 38,39] procedure of classical statistical mechanics which was later introduced to quantum statistics by Bogoliubov [4], we assume that all characteristic features of the quantum statistical state $\rho(t)$ can be inferred from expectation values of a certain, restricted set of operators $\left\{\hat{\gamma}_{i} \mid i \in \mathcal{I}\right\}$, where the index $i$ enumerates linearly independent operators $\hat{\gamma}_{i}$ from a (possibly infinite) index set $\mathcal{I}$.

Thus, we introduce a second coarse-grained, Markovian density operator $\sigma_{\left\{\gamma_{i}(t) \mid i \in \mathcal{I}\right\}}$ that approximates the complete density operator $\rho(t)$

$$
\sigma_{\left\{\gamma_{i}(t) \mid i \in \mathcal{I}\right\}} \approx \rho(t)=e^{-i \widehat{H} t} \rho(0) e^{i \widehat{H} t}
$$

The set of expectations values $\left\{\gamma_{i}(t) \mid i \in \mathcal{I}\right\}$ that parameterize the coarse-grained density operator are found by a quantum average $\langle\ldots\rangle$ over all states of the many-particle configuration space

$$
\gamma_{i}(t)=\left\langle\hat{\gamma}_{i}\right\rangle=\operatorname{Tr}\left\{\hat{\gamma}_{i} \rho(t)\right\}=\operatorname{Tr}\left\{\hat{\gamma}_{i} \sigma_{\left\{\gamma_{j}(t) \mid j \in \mathcal{I}\right\}}\right\} .
$$

While the coarse-graining assumption, i.e., the restriction to a few selected variables, is a statistical statement, the Markovian postulate concerns the separation of the time scales that govern the processes of equilibration and the decorrelation of fluctuations. Within this limit, the temporal evolution of this coarse-grained statistical density operator $\sigma_{\left\{\gamma_{i}(t) \mid i \in \mathcal{I}\right\}}$ is solely governed by the motion of the set of expectation values $\left\{\gamma_{i}(t) \mid i \in \mathcal{I}\right\}$. Any time dependence of the matrix elements of $\sigma_{\{\gamma(t)\}}$ can be 
attributed to the evolution of this restricted set of operators $\left\{\hat{\gamma}_{i}\right\}$, thus no relevant intrinsic time dependence is unaccounted for *. Although, the set of operators is unspecified so far, it can include operators such as unity (1), number $(\hat{N})$, linear momentum $(\hat{P})$, angular momentum $(\hat{L})$, and energy $(\widehat{H})$. The larger the set of operators, the better the quality of any subsequent approximation.

The third relevant many-particle density operator is $\sigma_{\{\gamma\}}^{(0)}$. It serves as a reference distribution and describes a relaxed (but non-equilibrium) state of the gas between consecutive collision events. We define it to yield the same expectation values as $\sigma_{\{\gamma\}}$ on the restricted set of operators $\left\{\hat{\gamma}_{i}\right\}$. Thus, this reference distribution is given by

$$
\sigma_{\{\gamma\}}^{(0)}=\exp \left(\hat{\gamma}_{i} \Upsilon_{\{\gamma\}}^{i}\right),
$$

where repeated indices imply a summation over operators $\hat{\gamma}_{i}$ and their conjugate thermo-dynamic coordinates $\Upsilon_{\{\gamma\}}^{i}$. These coordinates are defined implicitly by

$$
\gamma_{i}=\operatorname{Tr}\left\{\hat{\gamma}_{i} \sigma_{\{\gamma\}}\right\}=\operatorname{Tr}\left\{\hat{\gamma}_{i} \sigma_{\{\gamma\}}^{(0)}\right\} .
$$

It is of interest to note that the reference state, as given by the exponentiated form in Eq. (田), is still of the most general form permitted by the principles of quantum mechanics, as long as the set of operators $\left\{\hat{\gamma}_{i}\right\}$ is complete.

This ansatz for a non-equilibrium coarse-grained statistical operator $\sigma_{\{\gamma\}}$ and a "self-adjusting" reference distribution $\sigma_{\{\gamma\}}^{(0)}$ is also used in various contexts of equilibrium thermo-dynamics. For example, from a finite order truncation of a quantum virial expansion [4], one obtains a coarse-grained approximation $\sigma_{\{\Omega, \mu, \beta\}}$ of the grand-canonical statistical density operator $\rho=$ $\exp (\Omega-\mu \hat{N}-\beta \widehat{H})$. The starting point of the iteration procedure is a reference distribution $\sigma_{\{\Omega, \mu, \beta\}}^{(0)}$ that matches the solution as closely as possible. In here, the conjugate thermo-dynamic coordinates $\{\Omega, \mu, \beta\}$ correspond to the observables: unity, number and total energy.

\section{B. Derivation of an integral equation}

To find the functional form of the postulated coarsegrained Markovian density operator $\sigma_{\{\gamma(t)\}}$, we use a "boot-strapping" method. First, by assuming a given, yet unknown solution of Liouville's equation

\footnotetext{
*In the absence of ambiguities, we simplify the notation of the set of operators or their corresponding expectation values by dropping the index set $\mathcal{I}$, the indexing label $i$, or even the time argument $t$, completely.
}

$$
\frac{d}{d t} \sigma_{\{\gamma(t)\}}=-i\left[\widehat{H}, \sigma_{\{\gamma(t)\}}\right],
$$

we can determine the dynamical evolution of the expectation values $\gamma_{i}(t)=\operatorname{Tr}\left\{\hat{\gamma}_{i} \sigma_{\{\gamma(t)\}}\right\}$ from

$$
\frac{d}{d t} \gamma_{i}(t)=i \operatorname{Tr}\left\{\left[\widehat{H}, \hat{\gamma}_{i}\right] \sigma_{\{\gamma(t)\}}\right\} .
$$

Second, to derive the functional form of $\sigma_{\{\gamma(t)\}}$, we now use Eq. (6), Eq. (7), and the fact that there is no explicit time dependence in $\sigma_{\{\gamma(t)\}}$. Thus, by partial differentiation one obtains the following equation

$$
\operatorname{Tr}\left\{\left[\widehat{H}, \hat{\gamma}_{i}\right] \sigma_{\{\gamma\}}\right\} \partial_{\gamma_{i}} \sigma_{\{\gamma\}}=-\left[\widehat{H}, \sigma_{\{\gamma\}}\right],
$$

where again, we have adopted the convention that repeated indices imply summation, unless stated otherwise. Moreover, by dropping the explicit time dependence of the reference point in phase-space $\left\{\gamma_{i}(t)\right\}$, one can consider this as a partial-differential equation with independent variables $\left\{\gamma_{i}\right\}$.

The total Hamilton operator $\widehat{H}$ that governs the evolution of a weakly interacting, dilute gas permits a partitioning of the energy into a free part $\widehat{H}^{(0)}$ and a presumably weak interaction $\widehat{H}^{(1)}$

$$
\widehat{H}=\widehat{H}^{(0)}+\widehat{H}^{(1)} .
$$

One could use the bare, single-particle energy $\widehat{H}^{(0)}$ that determines the free kinetic evolution of the gas as a starting point of a series expansion of the coarse-grained density operator in terms of the interaction strength. However, it is well known that the mean-field interaction will significantly affect the single-particle energies. Anticipating this, we will shift the expansion point $\widehat{H}^{(0)}$ by an as yet undetermined single-particle energy $\widehat{Q}_{\{\gamma\}}^{(1)}$ to a dressed energy

$$
\widehat{H}_{\{\gamma\}}^{(0)}=\widehat{H}^{(0)}+\widehat{Q}_{\{\gamma\}}^{(1)} .
$$

To conserve energy, we have to reduce the interaction energy by an equal amount

$$
\widehat{H}_{\{\gamma\}}^{(1)}=\widehat{H}^{(1)}-\widehat{Q}_{\{\gamma\}}^{(1)} .
$$

The shifted interaction energy $\widehat{H}_{\{\gamma\}}^{(1)}$ represents the fluctuations about the mean-field energy and will be considered as "weak", or in other words, that strong fluctuations are well separated in time. This procedure is analogous to the first order energy shift found with ordinary Schrödinger-Rayleigh perturbation theory. The explicit form of this single-particle renormalization energy $\widehat{Q}_{\{\gamma\}}^{(1)}$ which is of the same order as the interaction energy, will be determined in the course of this calculation.

By expanding Eq. (8) around the dressed singleparticle energy $\widehat{H}_{\{\gamma\}}^{(0)}$, one obtains 
$\operatorname{Tr}\left\{\left[\widehat{H}_{\{\gamma\}}^{(0)}, \hat{\gamma}_{i}\right] \sigma_{\{\gamma\}}\right\} \partial_{\gamma_{i}} \sigma_{\{\gamma\}}+\left[\widehat{H}_{\{\gamma\}}^{(0)}, \sigma_{\{\gamma\}}\right]=F_{\{\gamma\}}^{(1)}$,

where $F_{\{\gamma\}}^{(1)}$ is introduced for convenience to hold the remaining first order contributions

$F_{\{\gamma\}}^{(1)}=-\left[\widehat{H}_{\{\gamma\}}^{(1)}, \sigma_{\{\gamma\}}\right]-\operatorname{Tr}\left\{\left[\widehat{H}_{\{\gamma\}}^{(1)}, \hat{\gamma}_{i}\right] \sigma_{\{\gamma\}}\right\} \partial_{\gamma_{i}} \sigma_{\{\gamma\}}$.

There are two interesting points in considering Eq. (12). First, there is not one unique solution $\sigma_{\{\gamma\}}$, but solution manifolds that are labeled by the constants of motion. Thus, any particular solution has to be augmented with appropriate physical boundary conditions. Second, the structure of the commutator of the single-particle Hamiltonian $\widehat{H}_{\{\gamma\}}^{(0)}$ with the set of operators $\left\{\hat{\gamma}_{i}\right\}$ is an intrinsic property of the system. It is determined both by the particular physical configuration and the set of operators. If the set of operators is chosen appropriately, the commutator forms an algebra with structure constants $\mathcal{A}_{\{\gamma\}}$ defined by

$$
\left[\widehat{H}_{\{\gamma\}}^{(0)}, \hat{\gamma}_{i}\right]=\mathcal{A}_{\{\gamma\}_{i}{ }^{j}} \hat{\gamma}_{j} .
$$

If we substitute this algebraic closure relation into Eq. (12), one obtains

$$
\left(\mathcal{A}_{\{\gamma\}}{ }^{i j} \gamma_{j}\right) \partial_{\gamma_{i}} \sigma_{\{\gamma\}}+\left[\widehat{H}_{\{\gamma\}}^{(0)}, \sigma_{\{\gamma\}}\right]=F_{\{\gamma\}}^{(1)} .
$$

By the method of characteristics, one can transform this first order, inhomogeneous partial-differential equation into an equivalent integral equation. Although formally equivalent, the later method is advantageous as it leads naturally to a series expansion of the coarsegrained density operator by iteration (compare Dyson series [41]). The characteristic trajectories are parameterized curves $\left\{\bar{\gamma}_{i}(\tau ;\{\gamma\})\right\}$ along which the boundary value of the coarse-grained density operator is propagating in phase-space according to Eq. (15). They are defined by

$$
\begin{aligned}
\frac{d}{d \tau} \bar{\gamma}_{i}(\tau ;\{\gamma\}) & =i \mathcal{A}_{\{\bar{\gamma}(\tau ;\{\gamma\})\}_{i}}{ }^{j} \bar{\gamma}_{j}(\tau ;\{\gamma\}), \\
\bar{\gamma}_{i}(\tau=0 ;\{\gamma\}) & =\gamma_{i} .
\end{aligned}
$$

and the corresponding boundary conditions. Formally, the solution of the differential equation defines a map $K_{\{\gamma\}}(\tau)$ from regions in phase-space connected by the characteristics, i.e.,

$$
\bar{\gamma}_{i}(\tau ;\{\gamma\})=K_{\{\gamma\}}(\tau)_{i}{ }^{j} \gamma_{j} .
$$

This map is obtained from the equation defining the characteristics Eq. (16), i.e.,

$$
\begin{aligned}
\frac{d}{d \tau} K_{\{\gamma\}}(\tau)_{i}{ }^{j} & =i \mathcal{A}_{\{\bar{\gamma}(\tau ;\{\gamma\})\}_{i}}{ }^{l} K_{\{\gamma\}}(\tau)_{l}{ }^{j}, \\
K_{\{\gamma\}}(\tau=0) & =\mathbf{1} .
\end{aligned}
$$

As the coarse-grained density operator is transported along the characteristics from its boundary value to its final value, it also evolves freely according to Eq. (15). To account for this evolution, we also define a unitary propagator $\widehat{U}_{\{\gamma\}}^{(0)}(\tau)$ by

$$
\begin{aligned}
\frac{d}{d \tau} \widehat{U}_{\{\gamma\}}^{(0)}(\tau) & =-i \widehat{H}_{\{\bar{\gamma}(\tau ;\{\gamma\})\}}^{(0)} \widehat{U}_{\{\gamma\}}^{(0)}(\tau), \\
\widehat{U}_{\{\gamma\}}^{(0)}(\tau=0) & =\mathbf{1} .
\end{aligned}
$$

The solution for the inhomogeneous partial-differential equation Eq. (15) is now obtained from an interactionpicture representation of the density operator $\sigma_{\{\gamma\}}(\tau)$ that is defined by

$$
\sigma_{\{\gamma\}}(\tau)=\widehat{U}_{\{\gamma\}}^{(0)}(\tau) \sigma_{\{\bar{\gamma}(\tau ;\{\gamma\})\}} \widehat{U}_{\{\gamma\}}^{(0)}(\tau) .
$$

At $\tau=0$, it coincides with the Schrödinger-picture value

$$
\sigma_{\{\gamma\}}(\tau=0)=\sigma_{\{\gamma\}},
$$

and, if our reference distribution, as defined in Eq. (4) matches the input state in the remote past asymptotically, i.e.,

$$
\lim _{\tau \rightarrow-\infty} \sigma_{\{\bar{\gamma}(\tau ;\{\gamma\})\}} \approx \lim _{\tau \rightarrow-\infty} \sigma_{\{\bar{\gamma}(\tau ;\{\gamma\})\}}^{(0)},
$$

then we find for the initial condition that

$$
\lim _{\tau \rightarrow-\infty} \sigma_{\{\gamma\}}(\tau)=\sigma_{\{\gamma\}}^{(0)} .
$$

Finally, by differentiating the interaction-picture representation Eq. (23), the use of Eq. (15) and a subsequent integration subject to the boundary conditions given in Eqs. (24, 26), the integral equation for the coarse-grained density operator is obtained:

$$
\begin{aligned}
\sigma_{\{\gamma\}} & =\sigma_{\{\gamma\}}^{(0)}-i \int_{-\infty}^{0} d \tau e^{\eta \tau} \widehat{U}_{\{\gamma\}}^{(0)^{\dagger}}(\tau)\left(\left[\widehat{H}_{\{\gamma\}}^{(1)}, \sigma_{\{\gamma\}}\right]+\right. \\
& \left.+\operatorname{Tr}\left\{\left[\widehat{H}_{\{\gamma\}}^{(1)}, \hat{\gamma}_{i}\right] \sigma_{\{\gamma\}}\right\} \partial_{\gamma_{i}} \sigma_{\{\gamma\}}\right)_{\mid \bar{\gamma}(\tau ;\{\gamma\})} \widehat{U}_{\{\gamma\}}^{(0)}(\tau) .
\end{aligned}
$$

This expression links the interacting coarse-grained density operator to its non-interacting value by evaluating the integrand along its collision history. A regularizing function $\left(\eta \rightarrow 0_{+}\right)$has been introduced and suppresses higher order correlations, built up in individual collisional events.

\section{The limit of weakly interacting, dilute gases}

In the case of a weakly interacting system, we can seek the solution to the integral equation Eq. (27) in form a power series of the density operator. The expansion parameter is given by the interaction strength

$$
\sigma_{\{\gamma\}}=\sum_{l=0}^{\infty} \sigma_{\{\gamma\}}^{(l)} .
$$


If this series is inserted into the integral equation for the coarse-grained density operator, then one finds for the first order correction

$$
\begin{aligned}
\sigma_{\{\gamma\}}^{(1)} & =-i \int_{-\infty}^{0} d \tau e^{\eta \tau} \widehat{U}_{\{\gamma\}}^{(0)^{\dagger}}(\tau)\left(\left[\widehat{H}_{\{\gamma\}}^{(1)}, \sigma_{\{\gamma\}}^{(0)}\right]+\right. \\
& \left.+\operatorname{Tr}\left\{\left[\widehat{H}_{\{\gamma\}}^{(1)}, \hat{\gamma}_{i}\right] \sigma_{\{\gamma\}}^{(0)}\right\} \partial_{\gamma_{i}} \sigma_{\{\gamma\}}^{(0)}\right)_{\mid \bar{\gamma}(\tau ;\{\gamma\})} \widehat{U}_{\{\gamma\}}^{(0)}(\tau)
\end{aligned}
$$

We will show that the first two terms of the series Eq. 28) are sufficient to determine the kinetic equations for the expectation values of the set of operators $\left\{\hat{\gamma}_{i}\right\}$ including second order (collisional) contributions.

It is important to note that the integrand has to be evaluated along the trajectories to the past. However, it can be further simplified by using again the interactionpicture representation, as derived from Eqs. (14, 19,21). First, one notices that the interaction-picture representation of the reference distribution is constant, if evaluated along the characteristic trajectories. This is implied in its definition.

$$
\sigma_{\{\gamma\}}^{(0)}=\widehat{U}_{\{\gamma\}}^{(0)^{\dagger}}(\tau) \sigma_{\{\bar{\gamma}(\tau ;\{\gamma\})\}}^{(0)} \widehat{U}_{\{\gamma\}}^{(0)}(\tau)
$$

Second, one finds that in the interaction-picture the relevant operator $\hat{\gamma}_{i}(\tau ;\{\gamma\})$ can be expressed as a linear combination of Schrödinger-picture operators $\hat{\gamma}_{j}$

$$
\hat{\gamma}_{i}(\tau ;\{\gamma\})=\widehat{U}_{\{\gamma\}}^{(0)^{\dagger}}(\tau) \hat{\gamma}_{i} \widehat{U}_{\{\gamma\}}^{(0)}(\tau)=K_{\{\gamma\}}(\tau)_{i}{ }^{j} \hat{\gamma}_{j}
$$

Third, we define an interaction-picture Hamiltonian by

$$
\widehat{H}_{\{\gamma\}}^{(1)}(\tau)=\widehat{U}_{\{\gamma\}}^{(0)^{\dagger}}(\tau) \widehat{H}_{\{\bar{\gamma}(\tau ;\{\gamma\})\}}^{(1)} \widehat{U}_{\{\gamma\}}^{(0)}(\tau) .
$$

With these definitions, one can evaluate the integrand along the characteristic. Details of this calculation are outlined in Appendix A. Thus, within the limit of weak interactions and the Markov approximation, one obtains the first two contributions to the coarse-grained density operator as

$$
\begin{aligned}
\sigma_{\{\gamma\}} & =\sigma_{\{\gamma\}}^{(0)}-i \int_{-\infty}^{0} d \tau e^{\eta \tau}\left(\left[\widehat{H}_{\{\gamma\}}^{(1)}(\tau), \sigma_{\{\gamma\}}^{(0)}\right]+\right. \\
& \left.+\operatorname{Tr}\left\{\left[\widehat{H}_{\{\gamma\}}^{(1)}(\tau), \hat{\gamma}_{i}\right] \sigma_{\{\gamma\}}^{(0)}\right\} \partial_{\gamma_{i}} \sigma_{\{\gamma\}}^{(0)}\right)+\mathcal{O}[2]
\end{aligned}
$$

It is easy to show that within these approximations the coarse-grained density operator is Hermitian, and that it yields exactly the same expectation values as the reference distribution alone, as defined in Eq. (5).

\section{Quantum kinetic equations}

With this first order result for the coarse-grained density operator, we can now address the second part of our "boot-strapping" procedure: the kinetic evolution of an expectation value $\langle\hat{o}\rangle$ subject to this coarse-grained density operator. The kinetic equations are obtained by averaging Heisenberg's equation with the coarse-grained density operator, as given in Eq. (7). As we are interested in a power series expansion of the kinetic equations, again we decompose the total Hamilton operator $\widehat{H}$ and the coarse-grained density operator $\sigma_{\{\gamma\}}$ into its various contributions in terms of the interaction strength, i.e.,

$$
\begin{aligned}
\frac{d}{d t}\langle\hat{o}\rangle & =i \operatorname{Tr}\left\{[\widehat{H}, \hat{o}] \sigma_{\{\gamma(t)\}}\right\}= \\
& =i \operatorname{Tr}\left\{\left[\widehat{H}_{\{\gamma(t)\}}^{(0)}+\widehat{H}_{\{\gamma(t)\}}^{(1)}, \hat{o}\right] \sum_{l=0}^{\infty} \sigma_{\{\gamma(t)\}}^{(l)}\right\} .
\end{aligned}
$$

By grouping the individual terms, one finds

$$
\frac{d}{d t}\langle\hat{o}\rangle=\sum_{l=0}^{\infty} R_{\{\gamma(t)\}}^{(l)}[\hat{o}]+L_{\{\gamma(t)\}}^{(l+1)}[\hat{o}],
$$

where we have introduced linear Liouville operators

$$
\begin{aligned}
& R_{\{\gamma\}}^{(l \geq 0)}[\hat{o}]=i \operatorname{Tr}\left\{\left[\widehat{H}_{\{\gamma\}}^{(0)}, \hat{o}\right] \sigma_{\{\gamma\}}^{(l)}\right\}, \\
& L_{\{\gamma\}}^{(l>0)}[\hat{o}]=i \operatorname{Tr}\left\{\left[\widehat{H}_{\{\gamma\}}^{(1)}, \hat{o}\right] \sigma_{\{\gamma\}}^{(l-1)}\right\} .
\end{aligned}
$$

\section{General observables}

To obtain practically applicable approximations for the kinetic equation, we will truncate these series at low order, i.e., first or second order. In the case of a general operator $\hat{o}$ that can not be represented by a linear combination of relevant operators, i.e., $\hat{o} \notin \operatorname{Span}\left(\left\{\hat{\gamma}_{i} \mid i \in \mathcal{I}\right\}\right)$ this means that

$$
\begin{aligned}
\frac{d}{d t}\langle\hat{o}\rangle & =R_{\{\gamma(t)\}}^{(0)}[\hat{o}]+L_{\{\gamma(t)\}}^{(1)}[\hat{o}]+ \\
& +R_{\{\gamma(t)\}}^{(1)}[\hat{o}]+L_{\{\gamma(t)\}}^{(2)}[\hat{o}]+\mathcal{O}[2],
\end{aligned}
$$

which is correct up to first order. At first glance, it seems to be inconsistent to include also the second order contribution $L^{(2)}$ in this first order expression. However, a closer inspection shows that this particular kinetic equation Eq. (38) preserves all constants of motion. In other words, if the operator $\hat{o}$ is an exact symmetry

$$
[\widehat{H}, \hat{o}]=0 \text {, }
$$

then, according to Eq. (38), all initial averages are conserved

$$
\frac{d}{d t}\langle\hat{o}\rangle=0 .
$$

This is particularly interesting if we consider constants of motion related to number $\left(\widehat{N}, \widehat{N}^{2}, \ldots\right)$ and energy $\left(\widehat{H}, \widehat{H}^{2}, \ldots\right)$, as implemented in many recent BEC experiments. If the systems are prepared initially in one of the standard thermo-dynamical ensembles: micro-canonical $(\Delta N=0, \Delta E=0)$, canonical $(\Delta N=0, \Delta E)$, or grandcanonical $(\Delta N, \Delta E)$, then these properties are preserved in time. 


\section{Master variables}

In the previous section the kinetic evolution of a general operator was examined. Now, we focus on the set of relevant operators $\left\{\hat{\gamma}_{i}\right\}$. The kinetic equations for the corresponding expectation values $\left\{\gamma_{i}\right\}$ constitute a selfconsistent set of master-equations that determine the system's evolution. Again, we are only interested in a low order truncation of Eq. (35). Due to the requirement that the reference distribution yields the same expectation values as the unexpanded state Eqs. (5,14), all contributions of $R_{\{\gamma\}}^{(l>0)}\left[\hat{\gamma}_{i}\right]=0$ vanish identically. Thus, we find a quantum kinetic master-equation correct up to third order, i.e.,

$$
\frac{d}{d t} \gamma_{i}(t)=R_{\{\gamma(t)\}}^{(0)}\left[\hat{\gamma}_{i}\right]+L_{\{\gamma(t)\}}^{(1)}\left[\hat{\gamma}_{i}\right]+L_{\{\gamma(t)\}}^{(2)}\left[\hat{\gamma}_{i}\right]+\mathcal{O}[3]
$$

By using the explicit expression for the coarse-grained density matrix Eq. (33), we find the following quantumBoltzmann equation

$$
\begin{aligned}
& \frac{d}{d t} \gamma_{i}(t)=i \operatorname{Tr}\left\{\left[\widehat{H}, \hat{\gamma}_{i}\right] \sigma_{\{\gamma(t)\}}^{(0)}\right\}+ \\
& -\int_{-\infty}^{0} d \tau e^{\eta \tau} \operatorname{Tr}\left\{\sigma_{\{\gamma(t)\}}^{(0)}\left[\widehat{H}_{\{\gamma(t)\}}^{(1)}(\tau),\left[\widehat{H}_{\{\gamma(t)\}}^{(1)}(0), \hat{\gamma}_{i}\right]+\hat{\gamma}_{j}\left(i \partial_{\gamma_{j}} L_{\{\gamma(t)\}}^{(1)}\left[\hat{\gamma}_{i}\right]+\operatorname{Tr}\left\{\left[\partial_{\gamma_{j}} \widehat{H}_{\{\gamma(t)\}}^{(1)}(0), \hat{\gamma}_{i}\right] \sigma_{\{\gamma(t)\}}^{(0)}\right\}\right)\right]\right\}
\end{aligned}
$$

where $\widehat{H}_{\{\gamma\}}^{(1)}(\tau)$ is the interaction picture Hamiltonian as defined in Eq. (32).

There are two interesting features in Eq. (42): First, the coherent part depends only on the total Hamiltonian $\widehat{H}$. Eventually, this part will determine the evolution of the expectation values subject to the external trapping-, and the mean-field potentials. Consequently, all derived mean-field potentials are invariant with respect to a particular partitioning of the total energy $\widehat{H}$, as introduced by the renormalization potential $\widehat{Q}_{\{\gamma\}}^{(1)}$ (see. Eq. (100). Second, the kinetic equation is strictly local in time, i.e., Markovian. The validity criterion for this result is a correlation time of the energy fluctuations which is much shorter than the time scale upon which the expectation values evolve and this is the case for a weakly interacting dilute gas. 
III. KINETIC EQUATIONS FOR MEAN FIELDS, NORMAL AND ANOMALOUS FLUCTUATIONS

In the previous sections, we determined the form of a coarse-grained density operator as a functional of a certain set of mean values. In turn, the temporal evolution of the mean values is determined by the kinetic equations Eq. (42). In this section, we apply these general results to the particular situation of weakly interacting, low temperature bosonic gas. Thus, we have to determine the predominant energy contributions to the system Hamiltonian $\widehat{H}$, as well as, decide on a relevant set of operators $\left\{\hat{\gamma}_{i}\right\}$.

\section{A. The dynamical evolution}

In second quantization, the removal or addition of a particle from or to a position $\mathbf{x}$ is described by the action of quantum field operators $\hat{a}_{\mathbf{x}}, \hat{a}_{\mathbf{x}}^{\dagger}$ on the corresponding quantum state. As we are considering bosonic particles, these fields have to obey a commutation rule in order to comply with the symmetrization postulate:

$$
\delta(\mathbf{x}-\mathbf{y})=\left[\hat{a}_{\mathbf{x}}, \hat{a}_{\mathbf{y}}^{\dagger}\right] .
$$

So far, we have introduced the quantum field $\hat{a}$ in a position representation $\hat{a}_{\mathbf{x}}$. However, in performing actual calculations other representations are often more favorable, for example, bare-harmonic oscillator-, or selfconsistent Hartree-Fock states. Consequently, we will delay that choice and work with a yet unspecified basis that spans the same single-particle Hilbert space $\mathcal{H}=\operatorname{Span}\left(\left\{\left|q_{1}\right\rangle \mid q_{1} \in \mathcal{Q}\right\}\right)$. Here, the index set $\mathcal{Q}$ encompasses all possible single-particle quantum numbers triples $q_{1}$. In this generic basis, the removal of a particle from a position $\mathbf{x}$ transforms into

$$
\begin{aligned}
\hat{a}_{\mathbf{x}} & =\sum_{q_{1}}\left\langle\mathbf{x} \mid q_{1}\right\rangle \hat{a}_{q_{1}} \equiv\langle\mathbf{x} \mid 1\rangle \hat{a}_{1}, \\
\delta_{q_{1}, q_{2}} & =\left[\hat{a}_{q_{1}}, \hat{a}_{q_{2}}^{\dagger}\right] .
\end{aligned}
$$

Here, $\hat{a}_{q_{1}}$ denotes a bosonic operator that removes a particle from a general mode $\left|q_{1}\right\rangle$. To simplify the notation, we drop the name of the dummy summation variable, i.e., $\hat{a}_{q_{1}} \equiv \hat{a}_{1}$, as well as the summation symbol itself.

The temporal evolution of a weakly interacting bosonic gas is governed by a Hamilton operator that consists of a single-particle energy in the presence of the external trapping potential, a two-particle interaction potential, as well as higher order contributions. Within the dilute gas limit, we want to assume that these higher order contributions, mediated through three-body collisions, are unlikely to occur, and we disregard them. Thus, we assume a Hamilton operator of the following form:

$$
\widehat{H}=\widehat{H}^{(0)}+\widehat{H}^{(1)}=H^{(0)^{12}} \hat{a}_{1}^{\dagger} \hat{a}_{2}+\phi^{1234} \hat{a}_{1}^{\dagger} \hat{a}_{2}^{\dagger} \hat{a}_{3} \hat{a}_{4} .
$$

Here, $\widehat{H}^{(0)}$ denotes a single-particle Hamilton operator with matrix elements $H^{(0)^{12}}=\left\langle 1\left|\mathbf{p}^{2} /(2 m)+V_{\text {ext }}(\mathbf{x})\right| 2\right\rangle$. To be specific, we assume for convenience that the external trapping potential is an isotropic harmonic oscillator, i.e., $V_{\mathrm{ext}}(\mathbf{x})=m \omega^{2}\left(x^{2}+y^{2}+z^{2}\right) / 2$. In most of the present experiments, the two-body interaction potentials $V_{\text {bin }}\left(\mathbf{x}_{1}-\mathbf{x}_{2}\right)$ are repulsive, of short range, and are described by the two-particle matrix elements:

$$
\begin{aligned}
\phi^{1234} & =\frac{1}{2}(\mathcal{S})\left\langle 1\left|\otimes\left\langle 2\left|V_{\text {bin }}\left(\mathbf{x}_{1}-\mathbf{x}_{2}\right)\right| 3\right\rangle \otimes\right| 4\right\rangle, \\
\phi^{1234} & =\phi^{1243}=\phi^{2134}=\phi^{2143} .
\end{aligned}
$$

Only the symmetric part of the two-particle matrix element $\phi^{1234}$ is physically relevant. Therefore, we have explicitly $(\mathcal{S})$ symmetrized it. In the low kinetic energy range that we are interested in, s-wave scattering is the dominant two-particle scattering event 42 46]. Thus, by discarding all details of the two-particle potential, we can describe the interaction strength with a single parameter $V_{0}$ related to the scattering length $a_{\mathrm{s}}$ by $V_{0}=4 \pi \hbar^{2} a_{\mathrm{s}} / \mathrm{m}$. This limit corresponds to a singular interaction potential, i.e., $V_{\text {bin }}\left(\mathbf{x}_{1}, \mathbf{x}_{2}\right)=V_{0} \delta\left(\mathbf{x}_{1}-\mathbf{x}_{2}\right)$. In the case of this delta potential, one finds for the two-body matrix elements:

$$
\phi^{1234}=\frac{V_{0}}{2} \int_{-\infty}^{\infty} d^{3} \mathbf{x}\langle 1 \mid \mathbf{x}\rangle\langle 2 \mid \mathbf{x}\rangle\langle\mathbf{x} \mid 3\rangle\langle\mathbf{x} \mid 4\rangle .
$$

which need not be symmetrized, as they are symmetric already. However, considering the caveats that are related to the singular functional form of the two-particle potential [39], we will only rely on the existence and symmetry of the two-particle matrix elements as defined in Eq. (47).

It is interesting to note that the Hamilton operator Eq. (46) is more general than its intended use. In case of trapped atoms that have several internal electronic states, it is only necessary to combine all external and internal quantum numbers into the definition of a single particle state, i.e., $\left|q_{1}\right\rangle=\left|n_{1}, l_{1}, m_{1} ; F_{1}, M_{1}, \ldots\right\rangle$. This implies that all derived results also hold true for multicomponent systems, provided the matrix elements $H^{(0)}{ }_{12}$ and $\phi^{1234}$ are generalized accordingly (for example, double condensate mean-field equations in Refs. 47, 48])

The renormalization potential $\widehat{Q}_{\{\gamma\}}^{(1)}$ accounts for the mean-field shifted energies that affect the single-particle propagation between consecutive collision events. We have seen already in the previous section that the meanfield potentials, which result from a first order calculation Eq. (42), are invariant under the particular choice of a energy partitioning. Now, by anticipating the results of the first-order calculation, we do not just find a single mean-field potential, but several: i.e., one occurring in the equation for mean-fields, and a different one occurring in the equation for fluctuations. Furthermore, due to the inclusion of the anomalous fluctuations (see next section), we no longer have particle-number conserving mean-field potentials either. However, as we will 
use the particle-conserving part of the mean-field energies to determine the "best" single-particle basis, we will also choose a renormalization potential that is number conserving, i.e.,

$$
\widehat{Q}_{\{\gamma\}}^{(1)}=\phi^{1234} \hat{a}_{1}^{\dagger} Q_{\{\gamma\}}^{23} \hat{a}_{4}
$$

where $Q_{\{\gamma\}}^{23}$ are the matrix-elements (yet to be determined).

\section{B. The set of relevant operators}

The derivation of the kinetic equations is based on the premise that only a few fundamental variables determine the gas's evolution on a coarse-grained time scale. These quantities will serve as master variables and any single time observable is linked to them. It is an intricate question to determine a set of relevant variables from general grounds up, but we are guided by the following physical arguments:

In the case of kinetic temperatures well above the transition temperature, it is sufficient to consider only the redistribution of populations $f_{q_{1}}=\left\langle\hat{a}_{q_{1}}^{\dagger} \hat{a}_{q_{1}}\right\rangle$ within generic quantum levels $\left|q_{1}\right\rangle$. The quantum average is defined by Eq. (3).

However, as temperatures are lowered, the spatial extension of a single-particle wave function becomes comparable to the mean inter-particle distance. Thus, it will be necessary to consider spatial coherences as well, i.e., $f_{q_{1}, q_{2}}=\left\langle\hat{a}_{q_{2}}^{\dagger} \hat{a}_{q_{1}}\right\rangle$. Note, this is still a single-particle quantity.

On the other hand, the most salient and fascinating feature of Bose-Einstein condensation is the formation of a macroscopic many-particle mean-field $\alpha_{q_{1}}=\left\langle\hat{a}_{q_{1}}\right\rangle$. By now, this is an experimentally well established fact and to a large degree the mean-field is described by the Gross-Pitaevskii equation. Moreover, there are theoretical predictions [17,49] indicating that anomalous fluctuations play a significant role as well. Consequently, we will also consider anomalous averages $m_{q_{1}, q_{2}}=\left\langle\hat{a}_{q_{1}} \hat{a}_{q_{2}}\right\rangle$ as independent relevant variables.

Thus, guided by the forgoing arguments, we choose the set of relevant operators as

$$
\begin{aligned}
\left\{\hat{\gamma}_{i} \mid i \in \mathcal{I}\right\} & =\left\{\mathbf{1}, \hat{a}_{q_{1}}, \hat{a}_{q_{2}}^{\dagger},\right. \\
\hat{\tilde{f}}_{q_{1} q_{2}} & =\left(\hat{a}_{q_{2}}^{\dagger}-\alpha_{q_{2}}^{*}\right)\left(\hat{a}_{q_{1}}-\alpha_{q_{1}}\right), \\
\hat{\tilde{m}}_{q_{1} q_{2}} & =\left(\hat{a}_{q_{1}}-\alpha_{q_{1}}\right)\left(\hat{a}_{q_{2}}-\alpha_{q_{2}}\right), \\
\hat{\tilde{n}}_{q_{1} q_{2}} & \left.=\left(\hat{a}_{q_{1}}^{\dagger}-\alpha_{q_{1}}^{*}\right)\left(\hat{a}_{q_{2}}^{\dagger}-\alpha_{q_{2}}^{*}\right) \mid q_{1}, q_{2} \in \mathcal{Q}\right\} .
\end{aligned}
$$

and denote the corresponding expectation values $\gamma_{i}=$ $\operatorname{Tr}\left\{\hat{\gamma}_{i} \sigma_{\{\gamma\}}\right\}$ by

$$
\begin{aligned}
\left\{\gamma_{i} \mid i \in \mathcal{I}\right\}= & \left\{1, \alpha_{q_{1}}, \alpha_{q_{2}}^{*},\right. \\
& \left.\tilde{f}_{q_{1} q_{2}}, \widetilde{m}_{q_{1} q_{2}}, \widetilde{n}_{q_{1} q_{2}} \mid q_{1}, q_{2} \in \mathcal{Q}\right\}
\end{aligned}
$$

With this set of independent variables, i.e., the mean fields and the fluctuations around them, we can parameterize the reference distribution as

$$
\begin{aligned}
\sigma_{\left\{\alpha, \alpha^{*}, \tilde{f}, \tilde{m}, \tilde{n}\right\}}^{(0)}= & \exp \left(\Omega_{\{\tilde{f}, \tilde{m}, \tilde{n}\}}-\hat{\tilde{f}}_{12} \Upsilon_{\{\tilde{f}, \tilde{m}, \tilde{n}\}}^{12}+\right. \\
& \left.-\hat{\tilde{m}}_{12} \Lambda_{\{\tilde{f}, \tilde{m}, \tilde{n}\}}^{12}-\hat{\tilde{n}}_{12} \Lambda_{\{\tilde{f}, \tilde{m}, \tilde{n}\}}^{12^{*}}\right)
\end{aligned}
$$

Here, we have used the implicit summation convention described in Eq. (44). The conjugate thermo-dynamic coordinates $\{\Omega, \Upsilon, \Lambda\}$ are implicitly defined by the quantum averages

$$
\langle\hat{o}\rangle_{\left\{\alpha, \alpha^{*}, \tilde{f}, \tilde{m}, \tilde{n}\right\}}=\operatorname{Tr}\left\{\hat{o} \sigma_{\left\{\alpha, \alpha^{*}, \tilde{f}, \tilde{m}, \tilde{n}\right\}}^{(0)}\right\},
$$

that is

$$
\begin{array}{ll}
\langle\mathbf{1}\rangle_{\left\{\alpha, \alpha^{*}, \tilde{f}, \tilde{m}, \tilde{n}\right\}}=1, & \left\langle\hat{a}_{1}\right\rangle_{\left\{\alpha, \alpha^{*}, \tilde{f}, \tilde{m}, \tilde{n}\right\}}=\alpha_{1}, \\
\left\langle\hat{\tilde{f}}_{12}\right\rangle_{\left\{\alpha, \alpha^{*}, \tilde{f}, \tilde{m}, \tilde{n}\right\}}=\tilde{f}_{12}, & \left\langle\hat{\tilde{m}}_{12}\right\rangle_{\left\{\alpha, \alpha^{*}, \tilde{f}, \tilde{m}, \tilde{n}\right\}}=\widetilde{m}_{12},
\end{array}
$$

as well as their complex conjugates $\widetilde{n}_{12}=\widetilde{m}_{12}^{*}$. The average of any other multiple operator product, occurring during the evaluation of the kinetic equations, are greatly simplified by the Gaußian structure of the reference distribution. A set of factorization rules, known as Wick's theorem [37], can be derived and its main results are outlined in Appendix B.

\section{Renormalized master equations}

In this section we present the results of applying the kinetic master equations Eq. (42) to the set of relevant operators defined in Eq. (51). Within the limits of the physical approximations we have obtained a self-consistent set of equations for the mean-field amplitude $\alpha$ that generalizes the Gross-Pitaevskii equation, a quantum Boltzmann equation for the normal fluctuations (depletion) $\tilde{f}$ and the anomalous fluctuations $\widetilde{m}$. The large number of individual algebraic transformations $(\approx 10000)$ that are necessary to obtain the final result prohibits attempts to evaluate the collision terms manually. Therefore, we developed a symbolic algebra package that performs the required calculations. The presentation of the final results of this calculation is greatly simplified by introducing the following single-particle Hilbert-space vectors (co, contra-variant)

$$
\langle\hat{a}\rangle \equiv|\alpha\rangle=\alpha_{1}|1\rangle,\langle\hat{a}\rangle^{\dagger} \equiv\langle\alpha|=\alpha_{1}^{*}\langle 1|,
$$

normal operators [tensor rank $(1,1)$ ]

$$
\tilde{f}=\tilde{f}_{12}|1\rangle\left\langle 2\left|, f^{(c)}=\alpha_{2}^{*} \alpha_{1}\right| 1\right\rangle\langle 2|,
$$

pseudo operators [tensor rank $(2,0)$ ]

$$
\widetilde{m}=\widetilde{m}_{12}|1\rangle|2\rangle, m^{(c)}=\alpha_{2} \alpha_{1}|1\rangle|2\rangle,
$$

and their Hermitian conjugates $\widetilde{n}=\widetilde{m}^{\dagger}, n^{(c)}=m^{(c)^{\dagger}}$. 


\section{Mean fields}

For the macroscopic many-particle mean field $|\alpha\rangle$, we find

$$
\begin{aligned}
\frac{d}{d t}|\alpha\rangle= & -i\left(H^{(0)}+1 U_{f(c)}+2 U_{\tilde{f}}\right)|\alpha\rangle+ \\
& -i V_{\widetilde{m}} \angle\langle\alpha|+L_{\left\{\alpha, \alpha^{*}, \tilde{f}, \tilde{m}, \tilde{n}\right\}}^{(2)}[\hat{a}],
\end{aligned}
$$

with a collision term given by

$$
\begin{aligned}
L_{\left\{\alpha, \alpha^{*}, \tilde{f}, \tilde{m}, \tilde{n}\right\}}^{(2)}[\hat{a}] & =\left(\Gamma_{\tilde{f} \tilde{f}(1+\tilde{f})}-\Gamma_{(1+\tilde{f})(1+\tilde{f}) \tilde{f}}+\quad(60)\right. \\
& \left.+2 \Gamma_{\tilde{f} \tilde{m} \tilde{n}}-2 \Gamma_{(1+\tilde{f}) \tilde{m} \tilde{n}}\right)|\alpha\rangle+ \\
& +2\left(\Gamma_{\tilde{f} \tilde{m}(1+\tilde{f})}-\Gamma_{(1+\tilde{f}) \tilde{m} \tilde{f}}\right)\langle\langle\alpha| .
\end{aligned}
$$

First of all, the field evolves unitarily in the nonlinear hermitian Hamilton operator which consists of a free part $H^{(0)}$, determined by the external trapping potential and any other applied electro-magnetic fields. The second and third contribution are the collision induced mean field potentials, denoted by $U_{f(c)}$ and $U_{\tilde{f}}$. While the first of these potentials is proportional the mean field density itself, the second potential $U_{\tilde{f}}$ reflects the influence of the normal fluctuation upon the mean field. It is important to note the different weighting factors 1 and 2 multiplying the potentials. They arise from the different quantum statistical fluctuation properties of a c-number mean field and a normal single particle density. Exactly the same weighting factors are also found with the variational Hartree-Fock-Bogoliubov approach [17]. The mean field potential is defined in terms of the two-particle interaction matrix elements Eq. (47) and a single-particle density operator $f$ that can be either $f^{(c)}$ or $\tilde{f}$

$$
U_{f}=2 \phi^{12^{\prime} 3^{\prime} 4^{\prime}} f_{3^{\prime} 2^{\prime}}|1\rangle\left\langle 4^{\prime}\right| .
$$

Due to the Hermiticity of the two-particle interaction energy and the positivity of the single particle density, it is also self-adjoint if, i.e., $U_{f}=U_{f}^{\dagger}$. It is interesting to see that in the case of a delta-potential Eq. (49) and a scalar field (i.e., no internal degrees of freedom), the mean field potential reduces to the well known potential energy density that is proportional to the local mean field density

$$
\left\langle\mathbf{x}\left|U_{f^{(c)}}\right| \mathbf{y}\right\rangle=V_{0} \delta(\mathbf{x}-\mathbf{y})|\langle\mathbf{x} \mid \alpha\rangle|^{2} .
$$

The fourth linear collisional contribution is proportional to the anomalous coupling strength $V_{\widetilde{m}}$ contracted $(\angle)$ with the adjoint field $\langle\alpha|$. In general, we obtain the contraction of two tensor fields $A \angle B$, from a basis representation of the two fields and a subsequent contraction of the last index of $A$ with the last index of $B$.

\footnotetext{
${ }^{\dagger}$ This potential $U_{f}$ is not related to the single-particle propagator $\widehat{U}_{\{\gamma\}}^{(0)}(\tau)$ defined in Eq. (21)
}

This non-Hermitian coupling is in general mediated by an anomalous average $m$, and explicitly given by

$$
V_{m}=2 \phi^{12^{\prime} 3^{\prime} 4^{\prime}} m_{3^{\prime} 4^{\prime}}|1\rangle\left|2^{\prime}\right\rangle .
$$

In here, $m$ stands for any anomalous average, either $m^{(c)}$ or $\widetilde{m}$. From the definition of the anomalous coupling, it can be seen easily that $V_{m}=V_{m}^{\top}$ is symmetric.

All of the remaining terms in Eq. (60) are second order collisional contributions. They always appear in pairs where one term corresponds to an in-process while the sign reversed companion describes a loss out of the field. A closer inspection reveals that there are essentially four types of processes occurring, i.e., collision events that involve zero to three anomalous averages. Furthermore, one finds that a normal fluctuation $\tilde{f}$ on the in-side is always accompanied by a bosonically enhanced $(1+\tilde{f})$ on the out-side, and vice versa. On the other hand, whenever a mean field density $f^{(c)}$, an anomalous mean field density $m^{(c)}$, or an anomalous fluctuation $\widetilde{m}$ occurs in an in-process they appear unaltered on the out-process. This behavior is analogous to atomic transition rates described by the Einstein A- and B coefficients which can be attributed to stimulated absorption- and emission, as well as spontaneous emission processes. The fact that the mean-field is never bosonically enhanced supports the interpretation that the mean-field acts as a classical driving field.

In detail, these collisions operators are described by the following operators and pseudo-operators

$$
\begin{aligned}
& \Gamma_{f f f}=8 \phi^{12^{\prime} 3^{\prime} 4^{\prime}} \phi_{\eta}^{1^{\prime \prime} 2^{\prime \prime} 3^{\prime \prime} 4^{\prime \prime}} f_{3^{\prime} 1^{\prime \prime}} f_{4^{\prime} 2^{\prime \prime}} f_{4^{\prime \prime} 2^{\prime}}|1\rangle\left\langle 3^{\prime \prime}\right|, \\
& \Gamma_{f m f}=8 \phi^{12^{\prime} 3^{\prime} 4^{\prime} \phi_{\eta}^{\prime \prime} 2^{\prime \prime} 3^{\prime \prime} 4^{\prime \prime}} f_{3^{\prime} 1^{\prime \prime}} m_{4^{\prime} 3^{\prime \prime}} f_{4^{\prime \prime} 2^{\prime}}|1\rangle\left|2^{\prime \prime}\right\rangle, \\
& \Gamma_{f m n}=8 \phi^{12^{\prime} 3^{\prime} 4^{\prime}} \phi_{\eta}^{1^{\prime \prime} 2^{\prime \prime} 3^{\prime \prime} 4^{\prime \prime}} f_{3^{\prime} 1^{\prime \prime}} m_{4^{\prime} 3^{\prime \prime}} n_{2^{\prime \prime} 2^{\prime}}|1\rangle\left\langle 4^{\prime \prime}\right|, \\
& \Gamma_{m m n}=8 \phi^{12^{\prime} 3^{\prime} 4^{\prime}} \phi_{\eta}^{1^{\prime \prime} 2^{\prime \prime} 3^{\prime \prime} 4^{\prime \prime}} m_{3^{\prime} 4^{\prime \prime}} m_{4^{\prime} 3^{\prime \prime}} n_{2^{\prime \prime} 2^{\prime}}|1\rangle\left|1^{\prime \prime}\right\rangle .
\end{aligned}
$$

From the time average over the interaction picture Hamilton operator that appears in the kinetic equation Eq. (42), one obtains an approximately energy conserving two-particle matrix element.

$$
\begin{aligned}
& \phi_{\eta}^{1^{\prime \prime} 2^{\prime \prime} 3^{\prime \prime} 4^{\prime \prime}}(t)=\int_{-\infty}^{0} d \tau e^{\eta \tau} \phi^{1234} \times \\
& K_{\{\gamma(t)\}}^{\dagger}(\tau)^{1^{\prime \prime}}{ }_{1} K_{\{\gamma(t)\}}^{\dagger}(\tau)^{2^{\prime \prime}}{ }_{2} K_{\{\gamma(t)\}}(\tau)^{3^{\prime \prime}}{ }_{3} K_{\{\gamma(t)\}}(\tau)^{4^{\prime \prime}}{ }_{4} .
\end{aligned}
$$

The restricted propagator that has been used here is explicitly given by the time-ordered exponential

$$
K_{\{\gamma(t)\}}(\tau)=T e^{i \int_{\tau}^{0} d s\left(H^{(0)}+Q_{\{\bar{\gamma}(s ;\{\gamma(t)\})\}}\right.} .
$$

where we obtained the operators $H^{(0)}$ and $Q_{\{\gamma\}}$ from the matrix elements given in Eqs. (46,50).

To see qualitatively why $\phi_{\eta}^{1{ }^{\prime \prime}} 2^{\prime \prime} 3^{\prime \prime} 4^{\prime \prime}$ is essentially nonzero only on the energy-shell of thickness $\eta$, it is useful to represent the restricted propagator with respect to the eigen-states of 


$$
\left(H^{(0)}+Q_{\{\gamma(t)\}}\right)|1\rangle=\varepsilon_{1}(t)|1\rangle .
$$

By assuming that the energy levels change adiabatically slow, one obtains approximately

$$
\phi_{\eta}^{1^{1 \prime} 2^{\prime \prime} 3^{\prime \prime} 4^{\prime \prime}}=\phi^{1^{\prime \prime} 2^{\prime \prime} 3^{\prime \prime} 4^{\prime \prime}}\left(\pi \delta_{\eta}\left(\Delta_{1^{\prime \prime} 2^{\prime \prime} 3^{\prime \prime} 4^{\prime \prime}}\right)+i \mathcal{P}_{\eta} \frac{1}{\Delta_{1^{\prime \prime} 2^{\prime \prime} 3^{\prime \prime} 4^{\prime \prime}}}\right),
$$

which is non-zero only if the energy difference $\Delta_{1^{\prime \prime 2} 2^{\prime \prime} 3^{\prime \prime} 4^{\prime \prime}}=\varepsilon_{1^{\prime \prime}}(t)+\varepsilon_{2^{\prime \prime}}(t)-\varepsilon_{3^{\prime \prime}}(t)-\varepsilon_{4^{\prime \prime}}(t)$ is smaller than $\eta$.

$$
\lim _{\eta \rightarrow 0_{+}} \frac{1}{\eta-i \Delta}=\pi \delta_{\eta}(\Delta)+i \mathcal{P}_{\eta} \frac{1}{\Delta},
$$

This result is analogous to the second order Born-Markov approximation [35. 


\section{Normal fluctuations}

The kinetic equation for the normal fluctuations (depletion) generalizes the quantum-Boltzmann equation found in many textbooks [4, 37]

$$
\begin{aligned}
& \frac{d}{d t} \tilde{f}=-i\left[H^{(0)}+2 U_{f^{(c)}}+2 U_{\tilde{f}}, \tilde{f}\right]+ \\
& \quad-i V_{\left(m^{(c)}+\widetilde{m}\right)} \angle \widetilde{n}+i \widetilde{m} \angle V_{\left(m^{(c)}+\widetilde{m}\right)}^{\dagger}+L_{\left\{\alpha, \alpha^{*}, \tilde{f}, \widetilde{m}, \widetilde{n}\right\}}^{(2)}[70)
\end{aligned}
$$

with second order collisional contributions

$$
\begin{aligned}
& L_{\left\{\alpha, \alpha^{*}, \tilde{f}, \tilde{m}, \tilde{n}\right\}}^{(2)}[\hat{\tilde{f}}]=\left(\Gamma_{\tilde{f} \tilde{f}(1+\tilde{f})}+2 \Gamma_{f^{(c)} \tilde{f}(1+\tilde{f})}+\Gamma_{\tilde{f} \tilde{f} f^{(c)}}+2 \Gamma_{\tilde{f}\left(m^{(c)}+\tilde{m}\right) \tilde{n}}+2 \Gamma_{\tilde{f} \tilde{m} n^{(c)}}+2 \Gamma_{f^{(c)} \tilde{m} \tilde{n}}\right)(1+\tilde{f})+ \\
& \quad-\left(\Gamma_{(1+\tilde{f})(1+\tilde{f}) \tilde{f}}+2 \Gamma_{f^{(c)}(1+\tilde{f}) \tilde{f}}+\Gamma_{(1+\tilde{f})(1+\tilde{f}) f^{(c)}}+2 \Gamma_{(1+\tilde{f})\left(m^{(c)}+\tilde{m}\right) \tilde{n}}+2 \Gamma_{(1+\tilde{f}) \tilde{m} n^{(c)}}+2 \Gamma_{f^{(c)} \tilde{m} \tilde{n}}\right) \tilde{f}+ \\
& \quad+2\left(\Gamma_{\tilde{f}\left(m^{(c)}+\tilde{m}\right)(1+\tilde{f})}+\Gamma_{f^{(c)} \tilde{m}(1+\tilde{f})}+\Gamma_{\tilde{f} \tilde{m} f^{(c)}}-\Gamma_{(1+\tilde{f})\left(m^{(c)}+\tilde{m}\right) \tilde{f}}-\Gamma_{f^{(c)} \tilde{m} \tilde{f}}-\Gamma_{(1+\tilde{f}) \tilde{m} f^{(c)}}\right) \angle \tilde{n}+\text { h.c. }
\end{aligned}
$$

First, one finds a unitary evolution in the presence of the external trapping-, the mean-field-, and the normal potential. Both of the self-induced potentials, $U_{f^{(c)}}$ and $U_{\tilde{f}}$ are weighted by a common factor of 2 (compare [17]). This is in contrast to the weighting factors appearing the mean-field Hamilton operator Eq. (59). But again, this fact can be traced back to different quantum statistical properties of the mean-field and the fluctuations. Second, it can be seen that the anomalous coupling strength is now proportional to the total anomalous average, i.e., $m^{(c)}+\widetilde{m}$. Third, in the absence of any mean-fields or anomalous averages the second order contribution in Eq (71) reduces to the well known Boltzmann collision term

$$
\Gamma_{\tilde{f} \tilde{f}(1+\tilde{f})}(1+\tilde{f})-\Gamma_{(1+\tilde{f})(1+\tilde{f}) \tilde{f}} \tilde{f} .
$$

By further assuming that the normal fluctuations are predominantly diagonal in an energy eigen-basis defined by the non-linear Hamilton operator of Eq. (70) (ergodic hypothesis), one recovers the Bose-Einstein distribution as the stationary distribution of particles within the quantum levels. However, the presence of the mean-field, as well as the anomalous averages lead to additional collision processes that must not be ignored in general. Eventually, these processes will lead to a self-consistent equilibrium partition of particles between mean-fields, normaland anomalous fluctuations. While a detailed numerical self-consistent solution of the set of kinetic equations is still under investigation, it is important to see that the total particle number $\langle\hat{N}\rangle=\operatorname{Tr}\left\{f^{(c)}\right\}+\operatorname{Tr}\{\tilde{f}\}$ is always conserved [compare Eq. (40)]

$$
\frac{d}{d t}\langle\hat{N}\rangle=0
$$




\section{Anomalous fluctuations}

In contrast to the normal fluctuations, the anomalous fluctuations do not evolve unitarily but rather as a tensor of rank $(2,0)$. Both, left and right generators of the timeevolution are identical to the Hamilton operator of the normal fluctuations.

$$
\begin{gathered}
\frac{d}{d t} \tilde{m}=-i\left(H^{(0)}+2 U_{f^{(c)}}+2 U_{\tilde{f}}\right) \angle \tilde{m}-i \tilde{m} \angle\left(H^{(0)}+2 U_{f^{(c)}}+2 U_{\tilde{f}}\right)+ \\
-i V_{\left(m^{(c)}+\tilde{m}\right)} \angle(1+\tilde{f})-i \tilde{f} \angle V_{\left(m^{(c)}+\tilde{m}\right)}+L_{\left\{\alpha, \alpha^{*}, \tilde{f}, \tilde{m}, \tilde{n}\right\}}^{(2)}[\hat{\tilde{m}}] \\
L_{\left\{\alpha, \alpha^{*}, \tilde{f}, \tilde{m}, \tilde{n}\right\}}^{(2)}[\hat{\tilde{m}}]=\left(\Gamma_{\tilde{f} \tilde{f}(1+\tilde{f})}+\Gamma_{\tilde{f} \tilde{f} f^{(c)}}+2 \Gamma_{\tilde{f} f^{(c)}(1+\tilde{f})}+2 \Gamma_{\tilde{f} \tilde{m}\left(n^{(c)}+\tilde{n}\right)}+2 \Gamma_{\tilde{f} m^{(c)} \tilde{n}}\right) \angle \tilde{m}+ \\
-\left(\Gamma_{(1+\tilde{f})(1+\tilde{f}) \tilde{f}}+\Gamma_{(1+\tilde{f})(1+\tilde{f}) f^{(c)}}+2 \Gamma_{(1+\tilde{f}) f^{(c)} \tilde{f}}+2 \Gamma_{(1+\tilde{f}) \tilde{m}(n(c)+\tilde{n})}+2 \Gamma_{(1+\tilde{f}) m(c) \tilde{n}}\right) \angle \tilde{m}+ \\
+\left(2 \Gamma_{\tilde{f}\left(m^{(c)}+\tilde{m}\right)(1+\tilde{f})}+2 \Gamma_{\tilde{f} \tilde{m} f^{(c)}}+2 \Gamma_{f^{(c)} \tilde{m}(1+\tilde{f})}+\Gamma_{\tilde{m} \tilde{m}\left(n^{(c)}+\tilde{n}\right)}+2 \Gamma_{\tilde{m} m^{(c)} \tilde{n}}\right) \angle \tilde{f}+ \\
\left.-\left(2 \Gamma_{(1+\tilde{f})(m(c)}+\tilde{m}\right) \tilde{f}+2 \Gamma_{(1+\tilde{f}) \tilde{m} f^{(c)}}+2 \Gamma_{f^{(c)} \tilde{m} \tilde{f}}+\Gamma_{\tilde{m} \tilde{m}\left(n^{(c)}+\tilde{n}\right)}+2 \Gamma_{\tilde{m} m^{(c)} \tilde{n}}\right) \angle(1+\tilde{f})+\operatorname{transp}
\end{gathered}
$$

These three sets of master equations for the meanfield, the normal-, anomalous fluctuations constitute the main result of this article. They unify and generalize simpler equations which have been obtained previously also by other methods. However, so far, we have not discussed the physical implications that will arise from a self-consistent solution of these equations. Specifically, we need to determine the following problems: (I) the equilibrium distribution of particles partitioned between mean-field, normal-, and anomalous fluctuations; (II) the importance and quantitative size of anomalous fluctuations; (III) the collisional damping rates and second order energy-shifts; (IV) the response of the equilibrium system to weak external perturbations, i.e. the collective excitation frequencies via linear response theory; (V) critical phenomena occurring around the onset of condensation; or, for example, (VI) the dynamics of the growth of the condensed phase. 


\section{OUTLOOK}

In the previous section, we have enumerated several quantities that need to be determined and interesting paths along which detailed calculations could proceed. We believe that amongst these issues, it will be most crucial to address problems (I) and (II) around $T \approx 0$, first. On one hand, present-day experiments have established that the mean-field description yields good agreement. On the other hand, there are various approaches to the self-consistent equilibrium for normal and anomalous fluctuations and not all implications have been elucidated.

The standard route to investigate this problem is based on finite temperature calculations in the Hartree-FockBogoliubov description. Various schemes employing, for example, the quasi-static Popov approximation, or more dynamical methods that go beyond it (i.e. the collisionless regime) are being investigated by several research groups.

This present, non-equilibrium approach provides an alternate route to the stationary solution. In particular, we expect that the presence of a large condensed phase will lead to a strong correlation of the low energy part of the normal and anomalous fluctuations $(\approx 1-2$ times the chemical potential $\mu$ of the condensate), while the high energy tail will be mostly in detailed balance at some temperature $T$. However, such a macroscopic "polarization" of the low energy part of the fluctuations can not be described within a simple ergodic hypothesis, and therefore requires a full quantum treatment.

The main obstacle to overcome in numerically answering this problem is the unfavorable scaling law of the collision operators. From a simple operations count, one finds that there are $N^{8}$ summations involved if $N$ is the number of energy levels being considered.

This burden can be alleviated by being more specific, i.e. by postulating a completely isotropic situation for a single condensed phase, an isotropic trapping potential, a rotationally invariant initial condition, as well as a short-range central two-particle interaction. Within this simplified model, one can then decompose all involved operators in terms of angular momentum sub-manifolds (i.e. irreducible tensor sets and use of Wiger-Eckart theorem). This assumption makes the quantum mechanical treatment of the low energy region $(\mu \leq \varepsilon \leq 2 \mu, N \approx 10-20)$ feasible and will lead to a self-consistent equilibrium. A detailed numerical investigation is in progress and results will be reported.

\section{CONCLUSIONS}

In this article, we have revisited the Chapman-EnskogBogoliubov procedure of non-equilibrium statistical mechanics to describe the kinetic evolution of a condensed bosonic gas of atoms towards equilibrium. Within a second order Born-Markov approximation, we consider the collision dynamics of macroscopic mean-fields, normal fluctuations, and anomalous averages. In particular, we have obtained a coupled set of master equations for these quantities that encompass the Gross-Pitaevskii mean-field equation, as well as the quantum-Boltzmann equation for the normal fluctuations as limiting cases. The mean-field potentials that are obtained from a first order calculation are in agreement with the results of a variational Hartree-Fock-Bogoliubov calculation. Beyond these first order energy shifts, we obtain second order collisional energy shifts and damping rates that are bosonically enhanced. We expect our results to be valid when strong collisions are well separated in time and when the mean-field induced energy shifts may be neglected during a strong collision event [see Eqs. (A7), (A8)].

\section{ACKNOWLEDGMENTS}

We gratefully acknowledge stimulating discussions with P. Zoller, C. W. Gardiner, K. Burnett and M. L. Chiofalo.

\section{APPENDIX A:}

With the definitions for the interaction-picture representation, we can rewrite the commutator term of Eq. (29) as

$$
\begin{aligned}
& \widehat{U}_{\{\gamma\}}^{(0)}(\tau)\left[\widehat{H}_{\{\bar{\gamma}(\tau ;\{\gamma\})\}}^{(1)}, \sigma_{\{\bar{\gamma}(\tau ;\{\gamma\})\}}^{(0)}\right] \widehat{U}_{\{\gamma\}}^{(0)}(\tau)= \\
& =\left[\widehat{H}_{\{\gamma\}}^{(1)}(\tau), \sigma_{\{\gamma\}}^{(0)}\right] .
\end{aligned}
$$

The trace term evaluated along the trajectory simplifies to

$$
\begin{aligned}
& \operatorname{Tr}\left\{\left[\widehat{H}_{\{\bar{\gamma}(\tau ;\{\gamma\})\}}^{(1)}, \hat{\gamma}_{i}\right] \sigma_{\{\bar{\gamma}(\tau ;\{\gamma\})\}}^{(0)}\right\}= \\
& =K_{\{\gamma\}}(\tau)_{i}{ }^{j} \operatorname{Tr}\left\{\left[\widehat{H}_{\{\gamma\}}^{(1)}(\tau), \hat{\gamma}_{j}\right] \sigma_{\{\gamma\}}^{(0)}\right\} .
\end{aligned}
$$

Finally, the self-tuning term of the reference distribution gives

$$
\begin{aligned}
& \widehat{U}_{\{\gamma\}}^{(0)^{\dagger}}(\tau) \partial_{\gamma_{i}} \sigma_{\{\gamma\}}^{(0)} \widehat{U}_{\mid \bar{\gamma}(\tau ;\{\gamma\})}^{(0)}(\tau)= \\
& =\left(\frac{\partial \bar{\gamma}_{i}(\tau ;\{\gamma\})}{\partial \gamma_{l}}\right)^{-1}\left(\left[\widehat{D}_{\{\gamma\}}(\tau)_{l}, \sigma_{\{\gamma\}}^{(0)}\right]+\partial_{\gamma_{l}} \sigma_{\{\gamma\}}^{(0)}\right)
\end{aligned}
$$

where we introduce auxiliary operator valued vectors $\widehat{D}_{\{\gamma\}}(\tau)$ and matrix-valued coefficients, $S_{\{\gamma\}}(\tau)$ by

$$
\begin{aligned}
\widehat{D}_{\{\gamma\}}(\tau)_{i} & =\widehat{U}_{\{\gamma\}}^{(0)^{\dagger}}(\tau) \partial_{\gamma_{i}} \widehat{U}_{\{\gamma\}}^{(0)}(\tau) \\
S_{\{\gamma\}}(\tau)^{i j} & =\left(\frac{\partial \bar{\gamma}_{l}(\tau ;\{\gamma\})}{\partial \gamma_{i}}\right)^{-1} K_{\{\gamma\}}(\tau)_{l}^{j}
\end{aligned}
$$


If these results are put together, one finds for the first order correction of the coarse-grained statistical operator

$$
\begin{aligned}
\sigma_{\{\gamma\}}^{(1)}= & -i \int_{-\infty}^{0} d \tau e^{\eta \tau}\left(\left[\widehat{H}_{\{\gamma\}}^{(1)}(\tau), \sigma_{\{\gamma\}}^{(0)}\right]+\right. \\
+ & \left(\left[\widehat{D}_{\{\gamma\}}(\tau)_{i}, \sigma_{\{\gamma\}}^{(0)}\right]+\partial_{\gamma_{i}} \sigma_{\{\gamma\}}^{(0)}\right) S_{\{\gamma\}}(\tau)^{i j} \\
& \left.\operatorname{Tr}\left\{\left[\widehat{H}_{\{\gamma\}}^{(1)}(\tau), \hat{\gamma}_{j}\right] \sigma_{\{\gamma\}}^{(0)}\right\}\right) .
\end{aligned}
$$

This expression is formally equivalent to Eq. (29). However, a closer inspection of the $S$ and $\widehat{D}$ terms shows that they contain higher order energy corrections induced by renormalization energy $\widehat{Q}_{\{\gamma\}}^{(1)}$. In particular, from a short time Taylor-expansion one finds that

$$
\begin{aligned}
\widehat{D}_{\{\gamma\}}(\tau) & =0-i \mathcal{O}\left[\begin{array}{ll}
\tau & \partial_{\gamma_{i}} \widehat{Q}_{\{\gamma\}}^{(1)}
\end{array}\right] \\
S_{\{\gamma\}}(\tau)^{i j} & =\delta^{i j}-i \mathcal{O}\left[\begin{array}{ll}
\tau & \left.\gamma_{l} \partial_{\gamma_{j}} \mathcal{A}_{\{\gamma\}_{i}}{ }^{l}\right] .
\end{array}\right.
\end{aligned}
$$

Consequently, we will disregard the effect of the meanfield onto the temporal evolution of $\widehat{D}_{\{\gamma\}}(\tau)$ and $S_{\{\gamma\}}(\tau)$ during a strong collision event and replace them by their "bare" values attained in the absence of the mean-field shift.

\section{APPENDIX B: A GENERALIZED WICK'S THEOREM}

The Gaußian structure of the reference distribution Eq. (53) is particularly useful, as it permits the systematic application of Wick's theorem [37]. This is a set of rules to efficiently evaluate quantum averages for multiple operator products as

$$
\left\langle\hat{\psi}_{1} \hat{\psi}_{2} \ldots \hat{\psi}_{l}\right\rangle_{\left\{\alpha, \alpha^{*}, \tilde{f}, \tilde{m}, \tilde{n}\right\}} .
$$

In this average, for example, the operator $\hat{\psi}_{1}$ represents either an operator $\hat{a}_{1}$ or $\hat{a}_{1}^{\dagger}$.

First, the displacement rule shifts any operator $\hat{\psi}_{1}$ by its c-number expectation value $\psi_{1}$ which is either $\alpha_{1}$ or $\alpha_{1}^{*}$, and replaces the quantum average by an average that has zero mean values:

$$
\begin{aligned}
& \left\langle\hat{\psi}_{1} \hat{\psi}_{2} \ldots \hat{\psi}_{l}\right\rangle_{\left\{\alpha, \alpha^{*}, \tilde{f}, \tilde{m}, \tilde{n}\right\}}= \\
& =\left\langle\left(\hat{\psi}_{1}+\psi_{1}\right)\left(\hat{\psi}_{2}+\psi_{2}\right) \ldots\left(\hat{\psi}_{n}+\psi_{l}\right)\right\rangle_{\{0,0, \tilde{f}, \tilde{m}, \tilde{n}\}} .
\end{aligned}
$$

Second, after expanding the multiple products, one can discard all averages that involve an odd numbers of operators:

$$
\left\langle\hat{\psi}_{1} \hat{\psi}_{2} \ldots \hat{\psi}_{2 s+1}\right\rangle_{\{0,0, \tilde{f}, \tilde{m}, \tilde{n}\}}=0 .
$$

And third, for the remaining averages, one can use the Gaußian factorization rule:

$$
\begin{aligned}
& \left\langle\hat{\psi}_{1} \hat{\psi}_{2} \ldots \hat{\psi}_{2 s}\right\rangle_{\{0,0, \tilde{f}, \tilde{m}, \tilde{n}\}}= \\
& =\left\langle\hat{\psi}_{1} \hat{\psi}_{2}\right\rangle_{\{0,0, \tilde{f}, \tilde{m}, \tilde{n}\}}\left\langle\hat{\psi}_{3} \ldots \hat{\psi}_{2 s}\right\rangle_{\{0,0, \tilde{f}, \tilde{m}, \tilde{n}\}}+ \\
& +\left\langle\hat{\psi}_{1} \hat{\psi}_{3}\right\rangle_{\{0,0, \tilde{f}, \tilde{m}, \tilde{n}\}}\left\langle\hat{\psi}_{2} \hat{\psi}_{4} \ldots \hat{\psi}_{2 s}\right\rangle_{\{0,0, \tilde{f}, \tilde{m}, \tilde{n}\}}+ \\
& \quad \quad \quad \\
& +\left\langle\hat{\psi}_{1} \hat{\psi}_{2 s}\right\rangle_{\{0,0, \tilde{f}, \tilde{m}, \tilde{n}\}}\left\langle\hat{\psi}_{2} \ldots \hat{\psi}_{2 s-1}\right\rangle_{\{0,0, \tilde{f}, \tilde{m}, \tilde{n}\}}
\end{aligned}
$$

By proceeding recursively, one has finally evaluated the complete multiple operator average Eq. (B1).

[1] M. H. Anderson, J. R. Ensher, M. R. Matthews, C. E. Wieman and E. A. Cornell, Science 269, 198 (1995).

[2] C. C. Bradley, C. A. Sackett, J. J. Tollett, and R. G. Hulet, Phys. Rev. Lett. 75, 1687 (1995).

[3] K. B. Davis, M.-O. Mewes, M. R. Andrews, N. J. van Druten, D. S. Durfee, D. M. Kurn and W. Ketterle, Phys. Rev. Lett. 75, 3969 (1995).

[4] A. I. Akhiezer and S. V. Peletminskii, Methods of Statistical Physics (Pergamon Press Ltd., Oxford, England, 1981).

[5] O. J. Luiten, M. W. Reynolds, and J. T. M. Walraven, Phys. Rev. A 53, 381 (1996).

[6] Y. Kagan, in Bose-Einstein Condensation, edited by A. Griffin, D. W. Snoke, and S. Stringari (Cambridge University Press, 1995), p. 202.

[7] N. P. Proukakis and K. Burnett, J. Res. Natl. Inst. Stand. Technol. 101, 457 (1996).

[8] M. Holland, J. Williams, K. Coakley, and J. Cooper, Quantum Semiclass. Opt. 8, 571 (1996).

[9] C. W. Gardiner and P. Zoller, Phys. Rev. A 55, 2902 (1997).

[10] D. Jaksch, C. W. Gardiner, and P. Zoller, Phys. Rev. A 56, 575 (1997).

[11] M. Holland, J. Williams, and J. Cooper, Phys. Rev. A 55, 3670 (1997).

[12] C. W. Gardiner, P. Zoller, R. J. Ballagh, and M. J. Davis, Phys. Rev. Lett. 79, 1793 (1997).

[13] C. W. Gardiner and P. Zoller, Phys. Rev. A 58, 536 (1998).

[14] D. Jaksch, C. W. Gardiner, K. M. Gheri, and P. Zoller, Phys. Rev. A 58, 1450 (1998).

[15] T. Lopez-Arias and A. Smerzi, Phys. Rev. A 58, 526 (1998).

[16] J. Javanainen, Phys. Rev. A 54, 3722 (1996).

[17] A. Griffin, Phys. Rev. B 53, 9341 (1996).

[18] D. A. W. Hutchinson, E. Zaremba, and A. Griffin, Phys. Rev. Lett. 78, 1842 (1997).

[19] F. Dalfovo et al., Phys. Rev. A 56, 3840 (1997).

[20] R. Dodd, M. Edwards, C. Clark, and K. Burnett, Phys. Rev. A 57, 32 (1998).

[21] A. Fetter and D. Rokhsar, Phys. Rev. A 57, 1191 (1998).

[22] S. Giorgini, Phys. Rev. A 57, 2949 (1998). 
[23] D. A. W. Hutchinson, R. J. Dodd, and K. Burnett, Phys. Rev. Lett. 11, 2198 (1998).

[24] P. O. Fedichev and G. V. Shlyapnikov, Phys. Rev. A 58, 3146 (1998).

[25] M. Holzmann, P. Grüter, and F. Laloë, http://xxx.lanl.gov/ cond-mat/, 9809356 (1998).

[26] B. D. Esry, Phys. Rev. A 55, 1147 (1997).

[27] J. L. Bohn, B. D. Esry, and C. H. Greene, Phys. Rev. A 58, 584 (1998).

[28] M. Bijlsma and H. T. C. Stoof, Phys. Rev. A 54, 5085 (1996).

[29] D. S. Jin, J. R. Ensher, M. R. Matthews, C. E. Wieman, and E. A. Cornell, Phys. Rev. Lett. 77, 420 (1996).

[30] M.-O. Mewes, M. R. Andrews, N. J. van Druten, D. M. Kurn, D. S. Durfee, C. G. Townsend, and W. Ketterle, Phys. Rev. Lett. 77, 988 (1996).

[31] D. S. Jin et al., Phys. Rev. Lett. 78, 764 (1997).

[32] A. Parkins and D. Walls, Phys. Rep. 303, 2 (1998).

[33] F. Dalfovo, S. Giorgini, L. Pitaevskii, and S. Stringari, http://xxx.lanl.gov/ cond-mat/, 9806038 (1998).

[34] G. V. Shlyapnikov and J. T. M. Walraven, Theory of ultra-cold Gases, private comm., FOM, Institute for Atomic and Molecular Physics, Kruislaan 407,1098 SJ Amsterdam, The Netherlands (1998).

[35] C. W. Gardiner, Quantum Noise (Springer Verlag, Berlin, 1991).

[36] H. Carmichael, An Open Systems Approach to Quantum Optics, Lecture Notes in Physics: $m 18$ (Springer-Verlag, Berlin, 1991).

[37] D. Zubarev, V. Morozov, and G. Röpke, Statistical Mechanics of Nonequilibrium Processes (Akademie Verlag, Berlin, 1997), Vol. 1, Basic Concepts, Kinetic Theory.

[38] S. Chapman and T. G. Cowling, The Mathematical Theory of Non-Uniform Gases (Cambridge University Press, Cambridge, 1970).

[39] K. Huang, Statistical Mechanics (John Wiley \& Sons, Inc., New York, 1965).

[40] D. Zubarev, V. Morozov, and G. Röpke, Statistical Mechanics of Nonequilibrium Processes (Akademie Verlag, Berlin, 1997), Vol. 2, Relaxation and Hydrodynamic Processes.

[41] W. Greiner and J. Reinhardt, Field Quantization (Springer Verlag, Berlin, 1996).

[42] A. J. Moerdijk and B. J. Verhaar, Phys. Rev. Lett. 73, 518 (1994).

[43] N. R. Newbury, C. J. Myatt, and C. E. Wieman, Phys. Rev. A 51, 2680 (1995).

[44] J. R. Gardner et al., Phys. Rev. Lett. 74, 3764 (1995).

[45] H. M. J. M. Boesten et al., Phys. Rev. A 55, 636 (1997).

[46] P. S. Julienne, F. H. Mies, E. Tiesinga, and C. J. Williams, Phys. Rev. Lett. 78, 1880 (1997).

[47] B. D. Esry, C. H. Greene, J. Burke, and J. L. Bohn, Phys. Rev. Lett. 78, 3594 (1997).

[48] B. D. Esry and C. H. Greene, Phys. Rev. A 57, 1265 (1998).

[49] S. Giorgini, L. Pitaevskii, and S. Stringari, Phys. Rev. Lett. 80, 5040 (1998). 